\title{
An Agile Governance Method for Multi-tier Industrial Architecture
}

\author{
Juan $\mathrm{Li}^{1,2}$, Frédérique Biennier ${ }^{1,2}$, and Chirine Ghedira ${ }^{1,3}$ \\ ${ }^{1}$ Université de Lyon, CNRS \\ 2 INSA Lyon LIRIS, UMR5205, F-69621, France \\ ${ }^{3}$ Université Lyon 1, LIRIS, UMR5205, F-69621, France \\ \{juan.1i, frederique.biennier\} @insa-lyon.fr, \\ cghedira@liris.cnrs.fr
}

\begin{abstract}
To fit the ever-changing business context, developing large scale networked and collaborative strategies involve increasing both enterprise and information system agility and interoperability. At the same time, lean and six sigma theories have also been used in industries to improve the industrial process itself so that profitability, quality and reputation are increased. In order to achieve these goals, an efficient and comprehensive governance method is necessary. However, mostly existing governance methods are isolated, they do not allow a dynamic composition of monitoring services, and they lack of a converging Business Process Management and Service Oriented Architecture as a holistic ecosystem. To overcome these limits, this paper propose an agile governance method for multi-tier industrial architecture, this architecture can couple the IT service principles with the Lean-6 sigma theories. This comprehensive governance architecture could eliminate monitoring blind spot lead to eliminate waste and defects.
\end{abstract}

Keywords: Service-Oriented Industrial Governance, SOA, Lean Six Sigma.

\section{Introduction}

To fit the renewed globalised economical environment, enterprises have to develop new large scale networked and collaborative strategies, involve increasing both enterprise and information system agility and interoperability. This trend has been favored by the development of interoperable and rather agile IT technologies based on services leading to SOA-based information systems reorganization. At the same time, lean and six sigma theories have also been used in industries to improve the industrial process itself so that profitability, quality and reputation are increased. One of the key points of lean six sigma consists in monitoring each step of manufacturing process and business service information as well as avoiding blind spots for governance.

However, most of the existing industrial services architecture governance methods are rather "fixed" and lack agility, overall perspective governance as they have unilateral perspective, just focused on the service level or IT vision. They do not support efficiently dynamic collaborative organization. As these isolated governance systems 
do not allow a dynamic composition of monitoring services and these service strategies lacks (by now) of an efficient governance system coupling the different layers of this complex ecosystem (including business strategies, business/industrial/IT services, execution platforms and infrastructure means).

To overcome this limit, we propose a multi-layer industrial service governance method, introducing the motivation and background (section 2). After, section 3 presents our multi-tier governance architecture's. Lastly, section 4 presents discussion and further works.

\section{Background}

In order to manage the increasing complexity of information technology systems and to deliver maximum real business value, Enterprise Architectures (EA) have been developed more than 20 years [1]. An EA explains how all the information technology elements in an organization - systems, processes, organizations, and people - work together as a whole [2]. Enterprise Architecture Framework (EAF) defines how to organize the structure and views associated with an EA [3].

A well functioning EA Governance is necessary to achieve a successful IT organization. Appropriate governance methods enable IT to become a key differentiator in creating an agile, adoptable enterprise [4].

Weill, P. and Ross, J. have presented that even some organizations have noticed the importance of EA Governance, most of the EA Governance methods separate the IT governance from business-performance metrics. There is still a big gap between business requirement and IT technology capacity, and it is difficult to make them understand each other [5].

Consequently, with the evolution of technology and management, industrial organization commonly has three layers: business layer, service layer, IT infrastructure layer. Service layer is an abstract layer connects business layer and IT infrastructure layer. Service-Oriented Architecture (SOA) is becoming the architectural style of choice in many enterprises. One of SOA's greatest strengths is its enhanced flexibility of services. It enables agile business processes and loose couple with specific technology [6]. Figure 1 gives a high-level view of the various SOA layers [7].

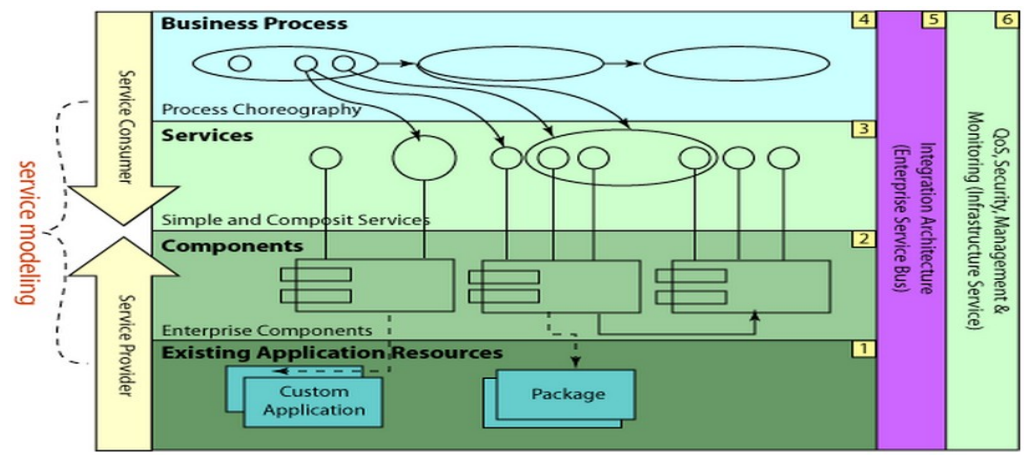

Fig. 1. High-level view of SOA layers (from Business-driven development IBM: http: / / www. ibm. com/developerworks / webservices/1ibrary/ws-bdd/) 
Due to the nature of SOA: demand for better business and IT alignment, the discipline of SOA governance has evolved. SOA governance defines the organizational structures and processes that are required to successfully adopt SOA, it helps minimize complications. SOA governance increases the connection among business processes, functional capabilities, organizational entities, end users, applications, and data. So SOA governance is a must if a company is to maintain and grow market share in a marketplace where customers expect and demand speed of delivery.

Numerous models for SOA Governance have been proposed so far. Michael N. and Julian E. et al. presented an approach for a generalized SOA Governance model. They identified six main components which form a mechanism for the optimal support of governance activities for an SOA system in a company. Nevertheless their SOA governance model, (as the most of SOA Governances) lack of ability to govern the IT infrastructure and ignore the infrastructure performance could impact the service performance [8]. Jan B. and Detlef S. outline a reference model for SOA governance that is based on the standardized SOA-RM [9] and motivated from aspects relevant to methodologies for SOA [10]. However, their model is conceptual and they did not propose approach to connect their model to common frameworks for IT governance and Enterprise Architecture. SOA Governance methods, cannot give a comprehensive perspective of industrial governance to combine the IT infrastructure ability with the business benefits. To face the challenge of improving competitiveness we need increase both enterprise and IT system agility and interoperability.

At a business layer, governance aims at managing business process, leading to BPM approaches. There is widespread usage of the terms Business Process Management (BPM) and SOA interchangeably. According to Gartner [11], Business Process Management "organizes people for greater agility," while SOA "organizes technology for greater agility." Business processes need to adapt to changes in the operating conditions and to meet the service-level agreements (SLAs) with a minimum of resources. According to Toyota case study, business processes hide inefficiencies. One has to follow the flow of information as the design evolves into the finished product [12]. Another good example of the benefits of BPM is the classic Ford case, from Hammer and Champy's seminal work [13].

With the recent economic turmoil, there is a trend of practically applying Lean 6 six sigma principles into industrial organization. This trend of combining six sigma quality with lean speed, which needs comprehensive governance methods to govern business, services and infrastructure performance, and clear interdependence of different layers' non-functional properties.

To overcome existing technological and organizational limits and lead the trend of utilizing Lean Six Sigma theory into collaborative industrial, we propose a multi-layer industrial service governance method to meet the ever-changing business requirements. It gives a global perspective of industrial governance, includes business level governance, service level governance and IT infrastructure governance. This comprehensive governance architecture could eliminate monitoring blind spot lead to eliminate waste and defects. With this governance method, industrial organization could achieve goals of Lean six sigma and their ultimate business requirements. 


\section{Contributions}

\subsection{Overview of Our Multi-tier Governance Architecture}

Our multi-tier governance architecture is designed to set a global governance environment to avoid any monitoring blind spot and could increase both enterprise and information system agility and interoperability, narrow the gap between business, service and infrastructure, reducing wastes and errors, enhancing the robustness of industrial multi-layer architecture and contribute to some commercial value.

We organize a global multi-tier governance architecture used to support both functional and non-functional management. Functional management deploys management engines to achieve functional requirements. Non-functional management cooperates with functional management and deploys performance indicators to evaluate performance of business processes.

To combine and orchestrate all the elements, we organize our multi-tier governance architecture as this way:

- A presentation Interface Layer (IL) to connect with users and to display governance results by customized dashboard.

- Platform Layers (PL) to achieve governance requirements. All management engines and performance indicators are orchestrated by customized agreements and governance rules. In this Platform Layers, we have 3 sub-levels to achieve business and governance requirements: (See figure 2) Business Level (BL) contains all of business context (business actors: deciders, clients and workers); Service Level (SL) is an abstraction level set between the BL and IT infrastructure. It includes all the components which are related to the services (service providers, service customers, service registry and middleware); and Application Level (AL) includes the entire infrastructures which should meet the needs of services (hardware, software, databases, firewalls, Intrusion prevention sys-tem, etc...). Management engines and indicators are deployed in

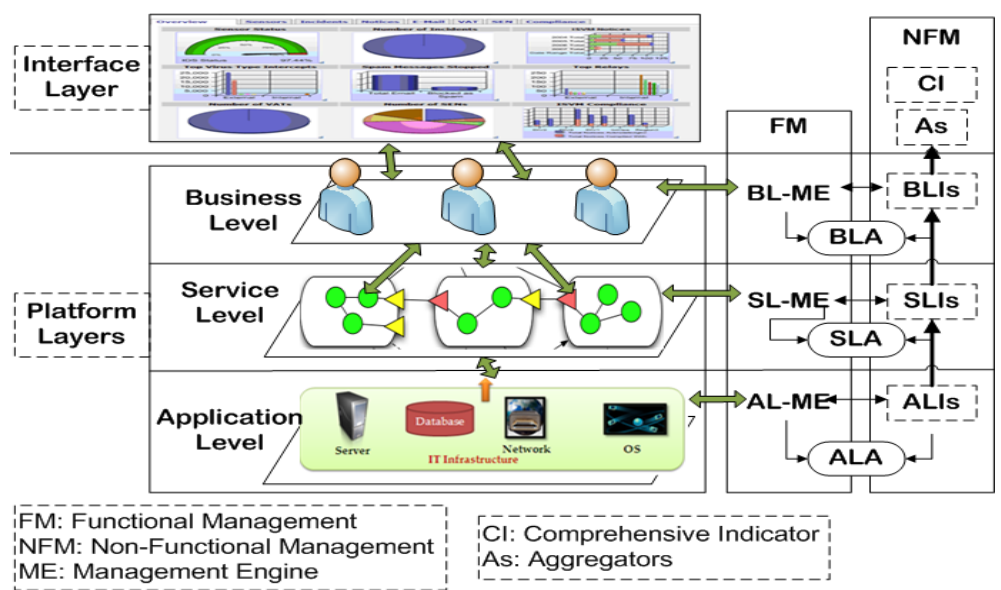

Fig. 2. Overview of Multi-tier Governance Architecture 
each level, Comprehensive Indicator (CI) and Aggregators combine scattered governance results from level indicators into comprehensive results for performance of business requirements.

Non-functional management transversal layer governs performance of functional management abide by agreements. Agreements are set at each level (BLA, SLA, ALA) to standardize non-functional constraints and to constrain the business value flow through entire enterprises.

- BLA as a guide to comply with the Business Quality Standards which could assist companies control quality (include manufacture quality and service quality) and maintain a high standard of customer satisfaction. BLA keeps the two key principles for company: "fit for purpose-the product and service should be suitable for the intended purpose; and right first time-mistakes should be eliminated."

- SLA in our multi-tier governance architecture is beyond the normal SLA in SOA. Our SLA is not only a negotiated agreement between service customers and extern service providers, but also it is the evaluation criteria for quality of service within our multi-tier governance architecture.

- ALA lists the expected functional and non-functional properties in application level. It set out the policies, strategies, specifications and criterion of application level. ALA defines all acceptable performances of infrastructure. It plays an important role for Application level indicators to measure performance of applications in this layer.

\subsection{Non-functional Governance Working Principles}

In order to make use of Lean 6 sigma theory to increase business efficiency, we should make sure every operation can add value to end customers, and we should eliminate any extra steps which cannot create profit to enterprise, the value flow and working operation steps of enterprise (from input raw material to distribute outputs through manufacture factor, must be taken into account).

Attributes of Functional Requirements and Non-functional Requirements: Functional Requirements (FRs) satisfy clients' business requirements; each 'business requirement' is completed by series Tasks; each 'task' is completed by some 'actions' at each level. Non-Functional Requirements (NFRs) constrain FRs. NFRs are divided into different NFRs-Families and NFRs-classes, according to the feature of NFRs. "FR", "action" and "NFR" could be defined and identified by their attributes. "Taskstamp" is synchronized with current task. "Level mark" labels a certain level. "Family mark" identifies NFPs belong to specific NFRs-Family and "class mark" identifies NFPs belong to specific classes. Therefore "family mark" and "class mark" are flags to automatically link relevant NFPs cross this multi-tier architecture. (Fig. 3) 


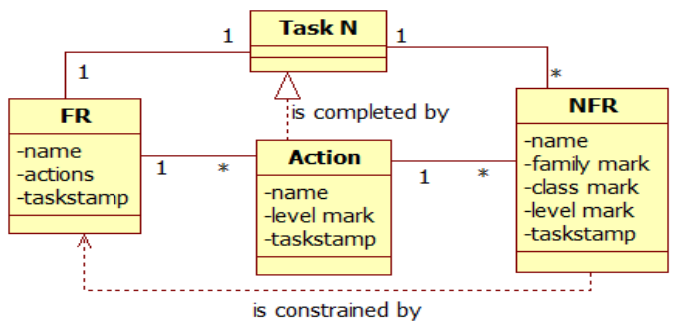

Fig. 3. Relation and Attributes of FRs, NFRs, Tasks and Actions

In order to make non-functional governance strategy convenient to retrospect governance situation and expediential to locate and correct mistakes, we take each "task" as a unit to govern and to give report. For each task, each level-indicator provides the real-time level governance report. Aggregators according to NFRs' feature to combine relevant scattered of level governance results to aggregate comprehensive governance results. After that the CI integrates and analyzes aggregators' reports, provides a comprehensive report to users by dashboard. Users can modify the way dashboard shows the governance results, such as users can choose charts or data to display timely governance results. As well as, dashboard can be customized to display level governance results or comprehensive business performance. All governance reports are business readable and non-programming.

Figure 4 gives us the horizontal and vertical view of this non-functional governance architecture's working principles. We can see that this non-functional governance architecture monitor completion performance of each task horizontally, and it monitor the completion performance of entire business requirement vertically.

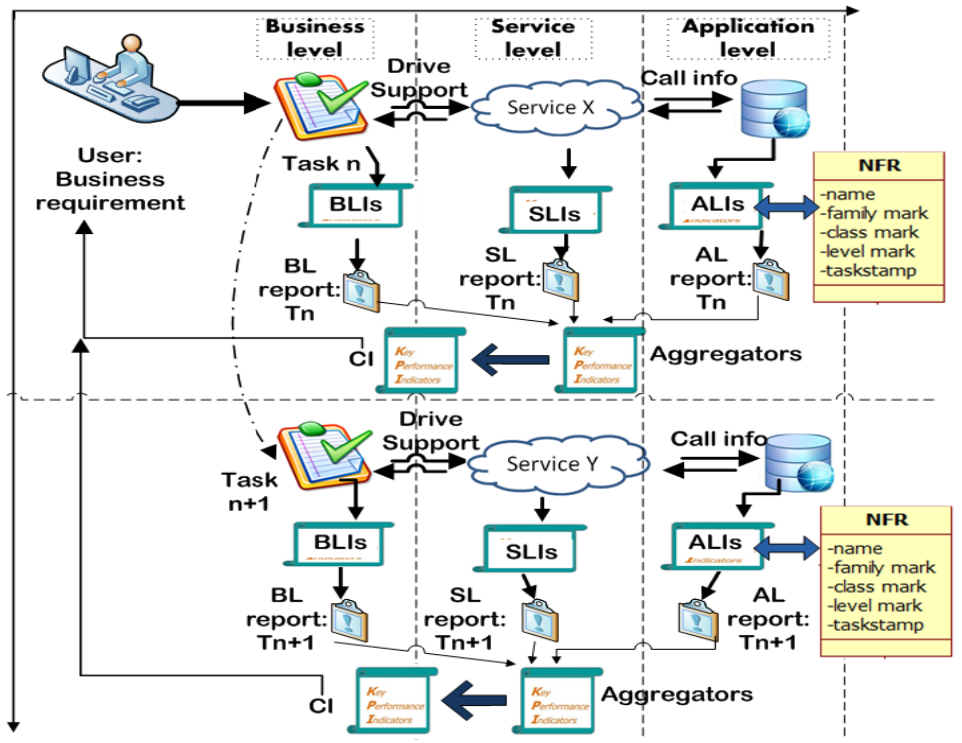

Fig. 4. Details of Horizontal and Vertical view of Governance 
The combination strategy of BLI, SLI and ALI plays an important role in this multi-tier architecture. According to the classification of NFRs, all of relevant NFRs cooperatively constrain FRs. Following picture gives us an example of NFRs' connection: NFRs in NFR-family (T) and NFR-class (RT and ET), these related NFRs could comprehensively constrain FRs cross three levels. (See figure 5)

This multi-tier governance architecture has ability of position precise governance point, and it has ability to evaluate any operation step could or not bring value to end users. It will improve agility of business and technologies. If there is any problem impedes value flow smoothly through entire enterprise, this architecture could find it out and fix it without impact other normal value flow steps.

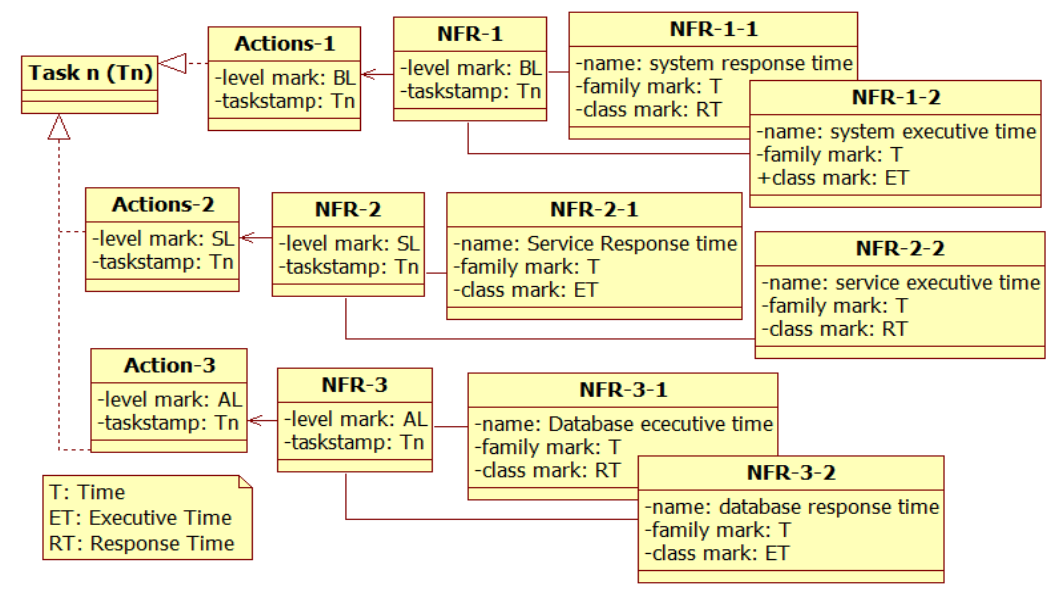

Fig. 5. Connection of Related NFRs

\section{Conclusion}

In this ever-changing economic environment, an ideal governance method could be significant for enterprises to achieve their business goals, to realize business and IT agility, to maximize their profits, to close the gap between business and IT, to eliminate wastes, to improve customer satisfaction, to enhance enterprises' comprehensive competitiveness, to win the future. According to the importance of governance architecture, we propose a multi-tier industrial governance architecture, which relies on the ability of linking dynamically industrial services in a customized industrial organization to fit the client requirements. This approach partly inspired by the Lean Six Sigma vision, extends the horizon of SOA to reveal a global vision, the enterprise business organization, abstract service network and its information infrastructure could be more agile.

Acknowledgments. This work extends the service showing strategy of the project process 2.0 granted by the DGCIS. 


\section{References}

1. Lankhorst, M., et al.: Enterprise Architecture at Work -Modeling, Communication, and Analysis. Springer, Heidelberg (2005)

2. Morganwalp, J.M., Sage, A.P.: Enterprise Architecture Measures of Effectiveness. International Journal of Technology, Policy and Management 4(1), 81-94 (2004)

3. Enterprise Architecture Framework, http://en.wikipedia.org/wiki/Enterprise_Architecture_framework

4. Infosys Technologies Limited whitepaper: EA: A Governance Framework (2005)

5. Weill, P., Ross, J.: A matrixed approach to designing IT governance. MIT Sloan Management Review 46(2) (2005)

6. Hitachi Consulting a knowledge-driven consulting whitepaper: SOA Governance: revolutionizing business to meet customer demands (2007)

7. Mitra, T.: Business-driven development, IBM, Software Group, http: / /www. ibm.com/developerworks / webservices/library/ ws-bdd/

8. Michael, N., Julian, E., Nicolas, R., Ralf, S.: Towards a Generic Governance Model for Service Oriented Architectures. In: Proceedings of AMCIS 2008 (2008), http://aisel.aisnet.org/amcis2008/361

9. MacKenzie, C.M., Laskey, K., McCabe, F., Brown, P.F., Metz, R.: OASIS Reference Model for Service Oriented Architecture 1.0 (October 2006)

10. Bernhardt, J., Seese, D.: A Conceptual Framework for the Governance of Service-Oriented Architectures. In: Feuerlicht, G., Lamersdorf, W. (eds.) ICSOC 2008. LNCS, vol. 5472, pp. 327-338. Springer, Heidelberg (2009)

11. Hill, J.B., Jim, S., David, F., Michael, J.M.: Gartner's position on business process management. In: Business Issues. Gartner, Inc. (2006)

12. Ryan, K.L.K.: A computer scientist's introductiory guide to business process management (BPM). In: Crossroads, vol. 15(4), ACM, NY (2009)

13. Michael, H.: Re-engineering work: Don't automate, obliterate. Harvard Business Review, 104-112 (1990) 